\title{
Mass and density determinations of 140 Siwa and 4979 Otawara as expected from the Rosetta flybys
}

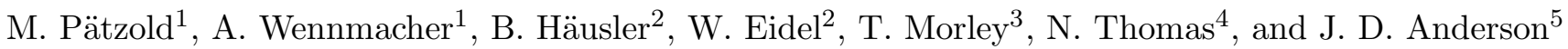 \\ 1 Institut für Geophysik und Meteorologie, Universität zu Köln, Albertus-Magnus-Platz, 50923 Köln, Germany \\ e-mail: wennmach@geo.Uni-Koeln.DE \\ 2 Institut für Raumfahrttechnik, Universität der Bundeswehr München, 85557 Neubiberg, Germany \\ e-mail: Bernd.Haeusler@UniBW-Muenchen.DE; werner.eidel@UniBW-Muenchen.DE \\ 3 ESA-ESOC, Robert-Bosch-Str. 5, 64293 Darmstadt, Germany \\ e-mail: tmorley@esoc.esa.de \\ 4 Max-Planck-Institut für Aeronomie, 37189 Katlenburg-Lindau, Germany \\ e-mail: thomas@linmpi.mpg.de \\ 5 Jet Propulsion Laboratory, Caltech, 4800 Oak Grove drive, Pasadena, CA 91107, USA \\ e-mail: John.D.Anderson@jpl.nasa.gov
}

Received 2 January 2001 / Accepted 13 February 2001

\begin{abstract}
During its interplanetary cruise to comet $\mathrm{P} /$ Wirtanen, the Rosetta spacecraft will encounter the asteroids 4979 Otawara and 140 Siwa on 11 July 2006 and 24 July 2008, respectively. The objective of the Rosetta Radio Science Investigations (RSI) experiment at these flybys is a determination of the asteroid's mass and bulk density by analyzing the radio tracking data (Doppler and range) received from Rosetta before, during and after closest approach. The spacecraft's flyby trajectory will be gravitationally deflected by an amount proportional to the mass of the asteroid for a given flyby distance and velocity. An analysis of the Doppler noise sources indicates that the mass can be determined to an accuracy of $1 \%$ for 140 Siwa. The corresponding bulk density show be accurate to $20 \%$. Unfortunately, a detectable trajectory perturbation seems to be hopeless for Otawara because of its small size and the large nominal flyby distance.
\end{abstract}

Key words. asteroids: mass - asteroids: individual: Siwa, Otawara

\section{Introduction}

The Rosetta spacecraft will be launched in January 2003 to intercept comet P/Wirtanen in 2011 and to escort the comet in an orbit about its nucleus from rendez-vous (about one year after the comet's aphelion) to its perihelion in July 2013. During its eight year long interplanetary cruise to its prime target, Rosetta will flyby at two main belt asteroids: 4979 Otawara on 11th July 2006 and 140 Siwa on 24th July 2008.

Apart from the cometary science objectives (Pätzold et al. 2000), the Radio Science Investigations (RSI) experiment onboard of Rosetta will also focus on the determination of the mass and density of the asteroids.

An asteroid's mass can be determined to a high degree of precision by analyzing spacecraft tracking data (Doppler frequency and range) received from a spacecraft flying by an asteroid. The gravitational attraction of the asteroid acting onto the spacecraft will influence the flyby

Send offprint requests to: M. Pätzold,

e-mail: paetzold@geo.Uni-Koeln.DE trajectory by an amount that is proportional to the asteroid's mass for a given flyby distance and velocity.

So far, the masses of only seven asteroids have been determined (see Yeomans et al. 1997 for a summary): four large main belt asteroids (1 Ceres, 2 Pallas, 4 Vesta and 11 Parthenope) from orbit perturbations and another three asteroids (243 Ida, 253 Mathilde and 433 Eros) from spacecraft encounters. The flybys of NEAR at Mathilde (Yeomans et al. 1997) and Eros (Yeomans et al. 1999, 2000) have provided the only mass determinations from spacecraft tracking. The mass of 243 Ida could only be constrained following the discovery of its moon Dactyl (Belton et al. 1995) from images from the Galileo solid state imager. Radio tracking was not sensitive enough to detect the slight change in Galileo's flyby trajectory because of the large flyby distance.

Bulk densities of asteroids are important parameters to understand their internal structure, any alteration during their life span and their origin.

Once the surface of the asteroid is mapped by imaging during the flyby, a shape model and the volume can be 
determined. The bulk density can then be estimated from the mass and volume.

\section{The Rosetta flyby at Siwa}

RSI will use the two-way radio carrier signals at $X$ band uplink $(7168 \mathrm{MHz})$ and $S$-band $(2300 \mathrm{MHz})$ and $X$-band ( $8422 \mathrm{MHz})$ simultaneous downlink, provided by the spacecraft radio subsystem, in order to measure slight changes in the spacecraft velocity induced by the gravity attraction of the asteroid via the classical Doppler effect. If the spacecraft's velocity is changed by forces acting on the spacecraft, a frequency shift of the radio carrier with respect to a predicted Doppler shift (assuming no perturbing body present) is induced by

$\Delta f=-f_{0} \frac{\Delta v_{r}}{c}$

where $\Delta v_{r}$ is the change of the spacecraft velocity along the line-of-sight (LOS) caused by non-accounted forces acting on the spacecraft, $f_{0}$ is the radio carrier frequency at $X$-band and $c$ is the speed of light. If the two-way radio link is used as described above, the Doppler shift is twice the value from (1).

Table 1 gives the parameters for both flybys and Fig. 1 sketches the flyby geometry for the Siwa case.

The flyby plane is defined as the plane containing the relative flyby velocity vector $\left(\boldsymbol{v}_{0}\right)$ and the direction vectors relative to the asteroid. The direction to the Sun lies also in this plane. During the flyby, the Rosetta spacecraft will point the instrument panel toward the asteroid. The spacecraft body will rotate during flyby in order to keep the remote sensing instruments pointed towards the asteroid. The fully steerable HGA will remain pointed toward the Earth which leads to a rotation of the HGA during flyby. A HGA rotation end point will be reached about five minutes before encounter resulting in the loss of radio signal. However, the instruments will remain pointed by the on-going rotation of the spacecraft body. The spacecraft is reoriented about $20 \mathrm{~min}$ after closest approach and spacecraft radio tracking will proceed after reestablishing the two-way radio link. The gravitational attraction from the asteroid acts onto the spacecraft at all times along the flyby trajectory (Fig. 1) leading to changes in the flyby velocity and trajectory. The deflection angle $\Psi$ is given by

$\operatorname{cosec} \frac{\Psi}{2}=1+\frac{r_{0} v_{0}^{2}}{G M}$

(Danby 1992), where $M=$ mass of the asteroid and $G=$ gravitational constant. For the nominal Siwa flyby at a closest approach distance of $r_{0}=3500 \mathrm{~km}$ and relative flyby velocity $v_{0}=17.04 \mathrm{~km} \mathrm{~s}^{-1}$, this angle is $10^{-5 \circ}$ and the trajectory deflection can be neglected. Therefore, we assume a straight flyby trajectory in the following with the radial distance vector (spacecraft to Siwa) of

$\boldsymbol{r}=\boldsymbol{r}_{0}+\boldsymbol{v}_{0}\left(t-t_{0}\right)$
The currently agreed nominal flyby distance of $3500 \mathrm{~km}$ is still subject to discussion among the Rosetta instrument teams and may change in the future. The smallest feasible flyby distance is $1750 \mathrm{~km}$ constrained by the maximum slew rate of the spacecraft. It is not expected that a flyby distance larger than $3500 \mathrm{~km}$ will finally be decided on.

Defining $\boldsymbol{g}(t)$ as the gravity attraction vector directed toward the asteroid at time $t$

$\boldsymbol{g}\left(t-t_{0}\right)=\frac{G M}{r^{2}} \boldsymbol{e}_{\mathrm{r}}$

where $t_{0}=$ time of closest approach. The vector can be resolved into two components along and across the trajectory direction. In the existing literature (Anderson 1971; Anderson \& Giampieri 1999; Rappaport et al. 2000) this is accomplished by integration over time with initial conditions at closest approach. Here, we choose to integrate with initial conditions at $-\infty$, which makes a small difference, equal to one-half of the total deflection angle (2), in the direction of the two components. The changes in velocity for both components because of the gravitational attraction are:

$$
\begin{aligned}
\Delta \boldsymbol{v}_{\text {along }}\left(t-t_{0}\right) & =\frac{G M}{r_{0} v_{0}} \frac{1}{\sqrt{1+\frac{v_{0}^{2}}{r_{0}^{2}}\left[t-t_{0}\right]^{2}}} e_{\mathrm{y}} \\
\Delta \boldsymbol{v}_{\text {across }}\left(t-t_{0}\right) & =-\frac{G M}{r_{0} v_{0}}\left[1+\frac{\frac{v_{0}}{r_{0}}\left[t-t_{0}\right]}{\sqrt{1+\frac{v_{0}^{2}}{r_{0}^{2}}\left[t-t_{0}\right]^{2}}}\right] \boldsymbol{e}_{\mathrm{x}} \cdot(5)
\end{aligned}
$$

Only the LOS components of these velocity changes can be observed via the classical Doppler effect. Relevant is the angle between the along-track direction and the projected LOS $\left(180^{\circ}-\alpha\right)$ and the angle between the across-track direction and the projected $\operatorname{LOS}\left(90^{\circ}-\alpha\right)$. Furthermore the Earth is located $\epsilon=3.35^{\circ}$ below the flyby plane. The total change in the Doppler velocity along LOS due to gravity attraction during the flyby is then

$$
\begin{aligned}
\Delta \boldsymbol{v}_{\mathrm{r}}= & -\Delta \boldsymbol{v}_{\text {along }} \cos \left(180^{\circ}-\alpha\right) \cos \epsilon \\
& +\Delta \boldsymbol{v}_{\text {across }} \cos \left(90^{\circ}-\alpha\right) \cos \epsilon \\
= & \Delta \boldsymbol{v}_{\text {along }} \cos \alpha \cos \epsilon+\Delta \boldsymbol{v}_{\text {across }} \sin \alpha \cos \epsilon
\end{aligned}
$$

The RSI flyby strategy is twofold. First, by comparing the predicted "force-free" pre-encounter Doppler frequency, gained from a precise orbit determination after the last manoeuvre some days before the encounter, with the actual observed Doppler frequency long after the closest approach but before the first manoeuvre after the flyby. This procedure for detecting velocity changes is similar to the one used for the Giotto flybys at comets P/Halley and P/Grigg-Skjellerup (Pätzold et al. 1991a, 1991b, 1993). The difference in Doppler frequency is translated into Doppler velocity along the LOS via (1), taking into account that the two-way radio link contributes twice the Doppler frequency value of (1), and, from the encounter geometry (Fig. 1), the asteroid's mass can be determined 
Table 1. Flyby parameters of the Rosetta asteroids

\begin{tabular}{lcc}
\hline \hline & Otawara & Siwa \\
\hline \hline Estimated diameter & $4 \mathrm{~km} \mathrm{(S} \mathrm{type)})^{a}$ & $110 \mathrm{~km}^{b}$ \\
& $2.8 \mathrm{~km}(\mathrm{~V} \text { type })^{a}$ & $\mathrm{~S}$ or V \\
Taxanomic type & $\geq 1900 \mathrm{~kg} / \mathrm{m}^{3}$ & $\mathrm{C}$ \\
Density & $2000 \mathrm{~kg} / \mathrm{m}^{3}$ \\
& $2183 \mathrm{~km}$ & $\begin{array}{c}\text { (estimate, used for simulation) } \\
\text { Nominal flyby distance }\end{array}$ \\
$\begin{array}{l}\text { Nominal flyby velocity } \\
\text { Angle between the flyby trajectory and } \\
\text { the projected line-of-sight to Earth }\end{array}$ & $10.63 \mathrm{~km} \mathrm{~s}^{-1}$ & $17.04 \mathrm{~km} \mathrm{~s}^{-1}$ \\
$\begin{array}{l}\text { Angle between the flyby plane and the } \\
\text { direction to Earth }\end{array}$ & $178.96^{\circ}$ & $174.04^{\circ}$ \\
$\begin{array}{l}\text { Angle between the flyby trajectory and } \\
\text { the direction to the Sun }\end{array}$ & $149.14^{\circ}$ & $3.35^{\circ}$ \\
\hline \hline
\end{tabular}

${ }^{a}$ Doressoundiram et al. (1999)

${ }^{b}$ M. Fulchignoni (priv. comm.)

\section{Rosetta flyby geometry at asteroid 140 Siwa}

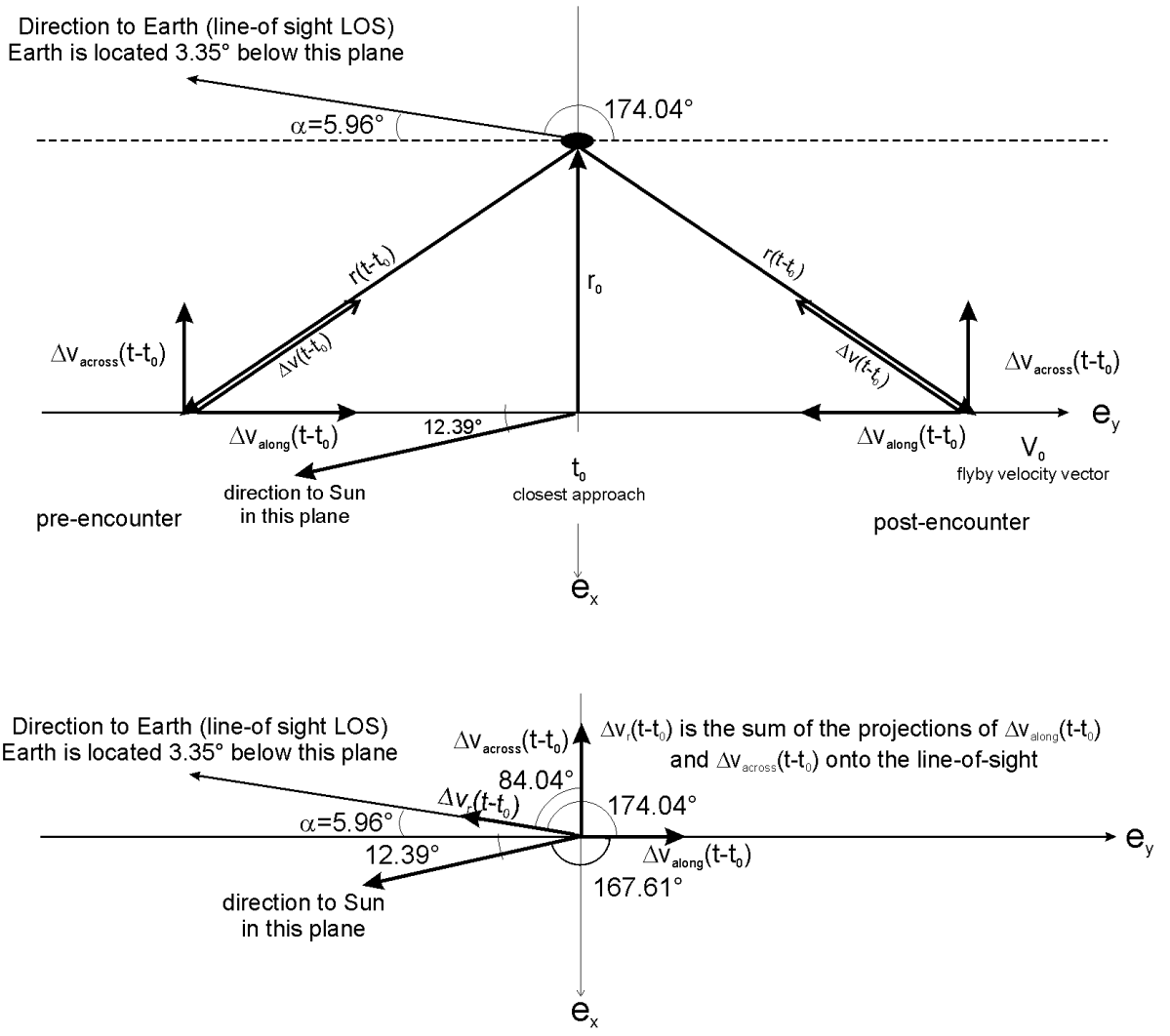

Fig. 1. Rosetta flyby geometry at asteroid 140 Siwa on 24th July 2008. The flyby plane contains the flyby trajectory $\left(\boldsymbol{v}_{0}\right)$ and the position vectors from spacecraft to asteroid. The projected direction to the Earth forms an angle of $174.04^{\circ}$ with the flyby trajectory. The direction to Earth is located $3.35^{\circ}$ below the plane. The change in velocity $\Delta v\left(t-t_{0}\right)$ can be split in two components along the track $\Delta v_{\text {along }}\left(t-t_{0}\right)$ and across the track $\Delta v_{\text {across }}\left(t-t_{0}\right)$. No net contribution to the total change in velocity is gained from the $\Delta v_{\text {along }}(t-$ $\left.t_{0}\right)$ component. Observed via the classical Doppler effect is $\Delta v_{r}\left(t-t_{0}\right)$, the sum of $\Delta v_{\text {along }}\left(t-t_{0}\right)$ and $\Delta v_{\text {across }}(t-$ $\left.t_{0}\right)$ projected onto the line-of-sight. The direction to the Sun lies in the flyby plane and forms an angle of $167.61^{\circ}$ with $\boldsymbol{v}_{0}$

from the absolute values

$$
\begin{aligned}
\Delta v_{\text {along }}(\infty) & =0 \\
\Delta v_{\text {across }}(\infty) & =2 \frac{G M}{r_{0} v_{0}} \\
\Delta v_{\mathrm{r}}(\infty) & =-\Delta v_{\text {accross }} \sin \alpha \cos \epsilon \\
& =-2 \frac{G M}{r_{0} v_{0}} \sin \alpha \cos \epsilon .
\end{aligned}
$$

Second, the real-time Doppler frequency is analysed during the flyby. Again,the Doppler velocity along the line- of-sight can be computed from (1) at any time $\left(t-t_{0}\right)$. Figure 2 shows the theoretical Doppler velocity signature for three different closest approach distances of $1750 \mathrm{~km}$ (the minimum feasible flyby distance), $3500 \mathrm{~km}$ (the nominal closest approach distance) and $10000 \mathrm{~km}$ for the Rosetta/Siwa flyby.

Figure 3 shows simulated noisy Doppler velocity measurements at 600 seconds integration time (solid circles) for the nominal flyby distance of $3500 \mathrm{~km}$. 


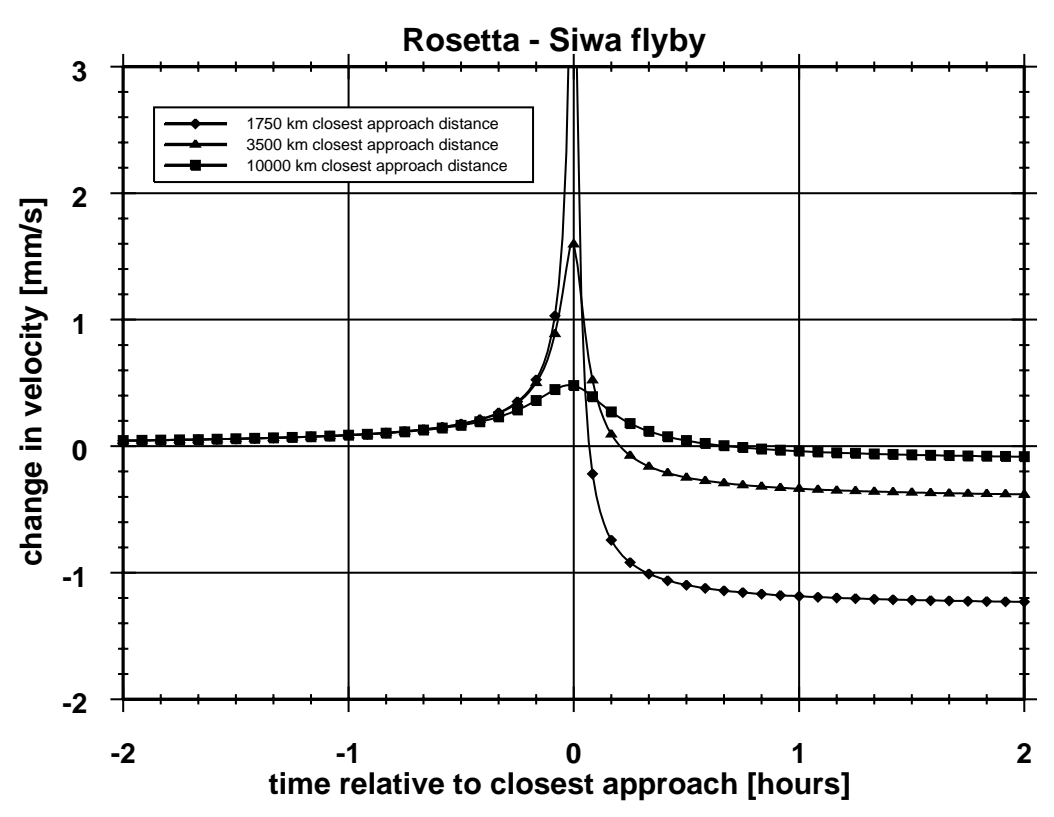

Fig. 2. Expected changes in velocity along the line-of-sight \pm 2 hours about the time of closest approach to the asteroid Siwa for three different flyby distances, $1750 \mathrm{~km}, 3500 \mathrm{~km}$ and $10000 \mathrm{~km}$
Table 2. Doppler velocity error budget

\begin{tabular}{|c|c|c|}
\hline & \multicolumn{2}{|c|}{ "Doppler velocity error $\sigma_{\mathrm{v}}$} \\
\hline & $S$-band & $X$-band \\
\hline $\begin{array}{l}\text { Phase error } \\
\text { (thermal and ground } \\
\text { station contribution }\end{array}$ & $1.0 \mathrm{~mm} / \mathrm{s}$ & $0.3 \mathrm{~mm} / \mathrm{s}$ \\
\hline $\begin{array}{l}\text { Transponder quantisation } \\
\text { error in frequency }\end{array}$ & $0.4 \mathrm{~mm} / \mathrm{s}$ & $0.1 \mathrm{~mm} / \mathrm{s}$ \\
\hline $\begin{array}{l}\text { Transponder quantisation } \\
\text { error in phase }\end{array}$ & $0.01 \mathrm{~mm} / \mathrm{s}$ & $0.004 \mathrm{~mm} / \mathrm{s}$ \\
\hline Total error (coherent mode) & $1.06 \mathrm{~mm} / \mathrm{s}$ & $0.32 \mathrm{~mm} / \mathrm{s}$ \\
\hline
\end{tabular}

Also included in the simulation is a gap of $25 \mathrm{~min}$ about closest approach (grey area) lasting from 5 min before closest approach to $20 \mathrm{~min}$ after closest approach due to the loss of the radio link. A non-linear least squares fit according to Eqs. (3) and (4) was applied to the simulated data points in order to retrieve an estimate for $G M$ of the asteroid.

\section{Doppler velocity accuracy}

Taking all major instrumental noise sources into account (spacecraft radio subsystem and ground station), the Doppler velocity error $\sigma_{v 0}$ for the Rosetta transponder system at $S$-band and $X$-band was estimated (Table 2 ) for a one second integration time. For longer integrations, the accuracy $\sigma_{v}(\Delta t)$ scales with the square root of the integration time $\Delta t$ :

$\sigma_{v}(\Delta t)=\sigma_{v 0} \frac{1}{\sqrt{\Delta t}}$

and is shown in Fig. 4.

Another uncertainty which adds to the Doppler noise is the propagation of the radio signals through the turbulent solar wind (Bird 1982; Pätzold et al. 2000). Turbulent solar wind density variations along the radio ray path will induce dispersive carrier phase shifts on the radio carrier, increasing the phase noise. Using a dual-frequency downlink at $S$-band and $X$-band, it is possible to limit the solar wind noise by extracting the dispersive propagation contributions from the calculation of the differential Doppler and by correcting the $X$-band two-way radio link by this result.

At the time of the Siwa flyby (24th July 2008), the Siwa-Sun-Earth angle is $100^{\circ}$ (Fig. 5). The impact parameter of the radio ray path along the Earth-Siwa radius is about 191 solar radii still close enough for contributions from the coronal plasma (solar wind) to the radio link.

We estimate an enhancement of the Doppler noise by a factor of two with respect to the instrumental noise.

The integration time which will be used during the flybys are $600 \mathrm{~s}$, yielding a theoretical $13 \mu \mathrm{m} / \mathrm{s} \mathrm{rms} \mathrm{noise} \mathrm{fig-}$ ure for the Doppler velocity from (8). The post-encounter Doppler velocity value for the $10000 \mathrm{~km}$ distance in Fig. 2 is $100 \mu \mathrm{m} / \mathrm{s}$, approximately four $\sigma$ above Doppler noise level and therefore clearly detectable assuming the increase in noise by a factor of two with respect to (8) because of the propagation in the solar wind. Anderson et al. (1992) estimated the fractional GM error as

$\frac{\sigma_{G M}}{G M}=\frac{v_{0} d}{G M} \sigma_{\mathrm{v}}$

where $\sigma_{\mathrm{v}}$ is the Doppler velocity noise. Applied to the simulation in Fig. 3, the fractional $G M$ error $\sigma_{G M}$ for the Siwa flyby is estimated to be in the order of $1 \%$. If the Sun proves quiet at the time of the flyby, this error might even be smaller. The direction to the Sun is located in the flyby plane and forms an angle of $167.61^{\circ}$ with $\boldsymbol{v}_{0}$. The radiation pressure acts in the anti-solar direction and forms an angle of $12.39^{\circ}$ leading to acceleration components along the track parallel to and across the track perpendicular to $\boldsymbol{v}_{0}$ (parallel to $\Delta \boldsymbol{v}_{\text {across }}$ ). During a ten hour tracking 


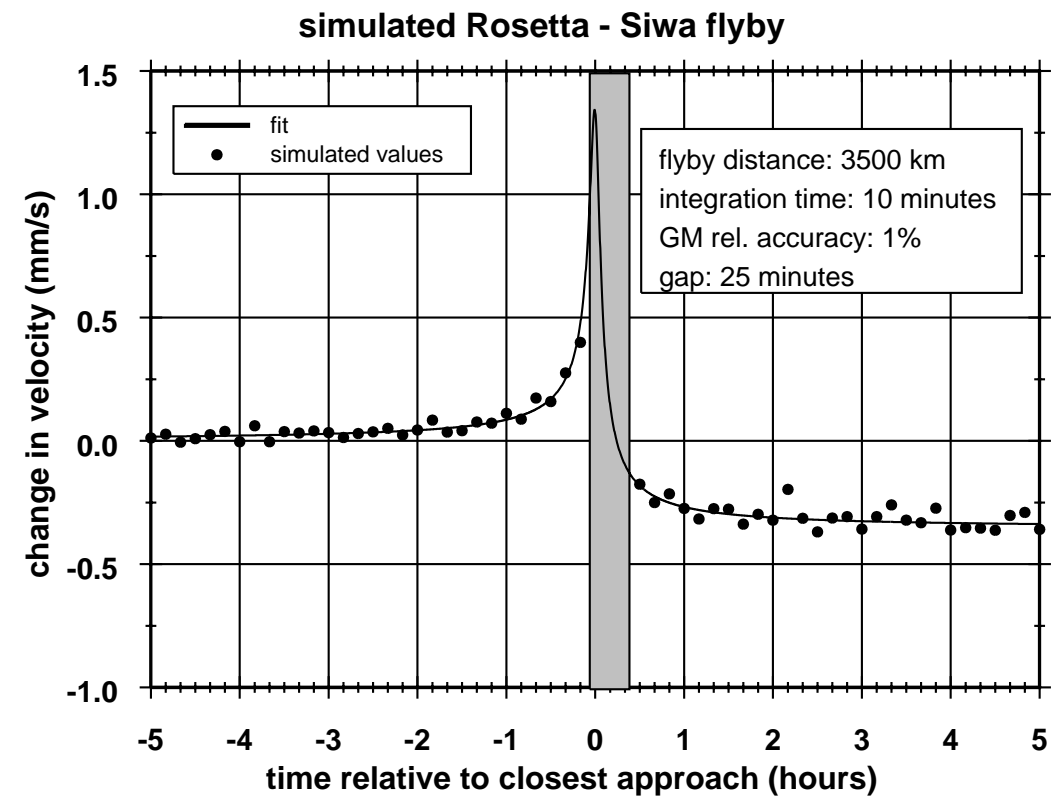

Total Doppler Noise

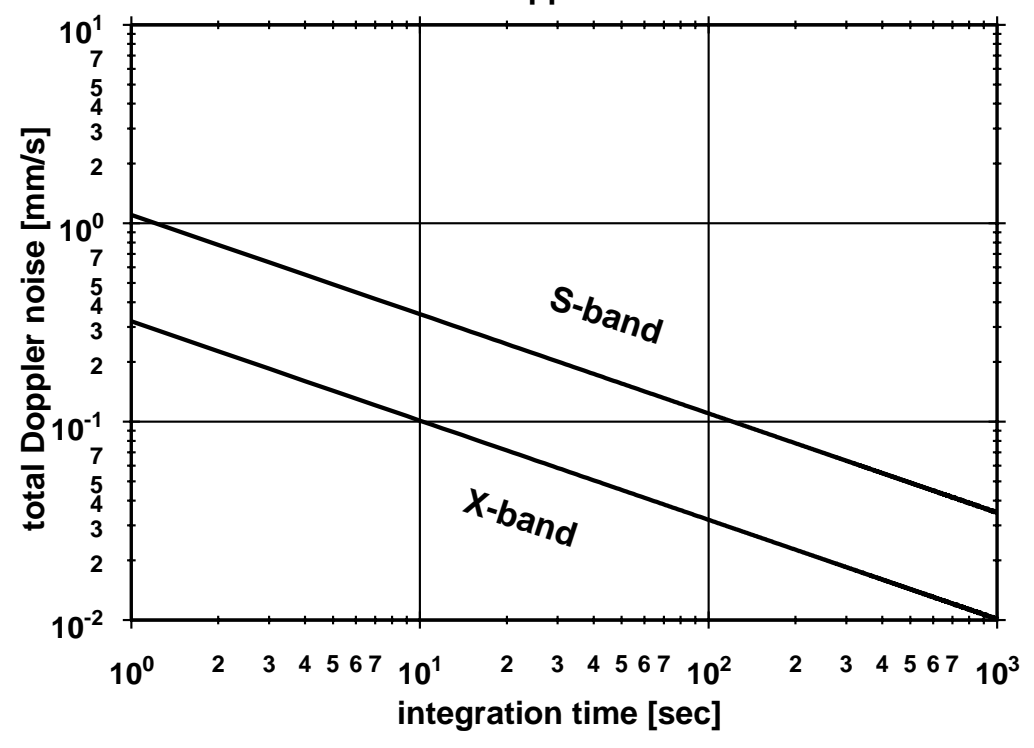

Fig. 3. Simulated noisy Doppler velocity values for an integration time of $600 \mathrm{~s}$ (solid points) according to Eqs. (5) and (6). A random Gaussian distributed noise of $\sigma_{\mathrm{v}}=15 \mu \mathrm{m} / \mathrm{s}$ was added to each simulated data point plus an grossly overestimated contribution of the same order of magnitude from the turbulent solar wind. The grey area marks 20 min of expected loss of radio signal. The solid line is an on-linear least squares fit to the simulated data points that yields $G M$ as a result to an accuracy of $1 \%$. The same accuracy was estimated from Anderson's (Anderson et al. 1992) rule of thumb (9)

Fig. 4. Estimated instrumental Doppler noise (from the radio subsystem and the ground station) at $S$-band (upper curve) and $X$-band (lower curve) as a function of integration time. Integration times of $600 \mathrm{~s}$ are planned to be used for the asteroid flybys

pass, the radiation pressure will induce a velocity component along the line-of-sight of $0.7 \mathrm{~mm} / \mathrm{s}$ (red shift), caused by a dominant velocity component parallel to $\boldsymbol{v}_{0}$. This is compared to the change in velocity by the gravitational attraction of the asteroid of $-0.3 \mathrm{~mm} / \mathrm{s}$ (Fig. 3) at the nominal flyby distance (blue shift). The radiation pressure contribution, depending on spacecraft mass, crosssectional area exposed to solar radiation, absorption and reflection capabilities of the spacecraft's surfaces and the heliocentric distance, can be predicted to a large extent and removed from the observed Doppler data. Errors in the prediction will appear as a linear trend in the observed Doppler residuals (observed minus predicted Doppler).

While the determination of the mass of Siwa from the RSI experiment is likely to have an accuracy of $1 \%$, the es- timate of the volume and hence the density will be much more inaccurate. The OSIRIS camera system on board Rosetta has avery high resolution of $60 \mathrm{~m} / \mathrm{px}$ at closest approach. Thus, in two dimensions, OSIRIS will determine the cross-sectional area of the target to about $0.5 \%$. However, the third dimension is unseen by the camera because it is unilluminated. Hence, this dimension can only be constrained from remote observations taken several hours before and using the asteroid's rotation. Even in this case, the rotation is unlikely to be orthogonal to the Sun-asteroid line (i.e. there is a constantly unilluminated pole). Thus, estimates, based on the asteroid having a reasonably continuous shape, have to be made. We estimate the resulting error to be of the order of $20 \%$ in both volume and density. 


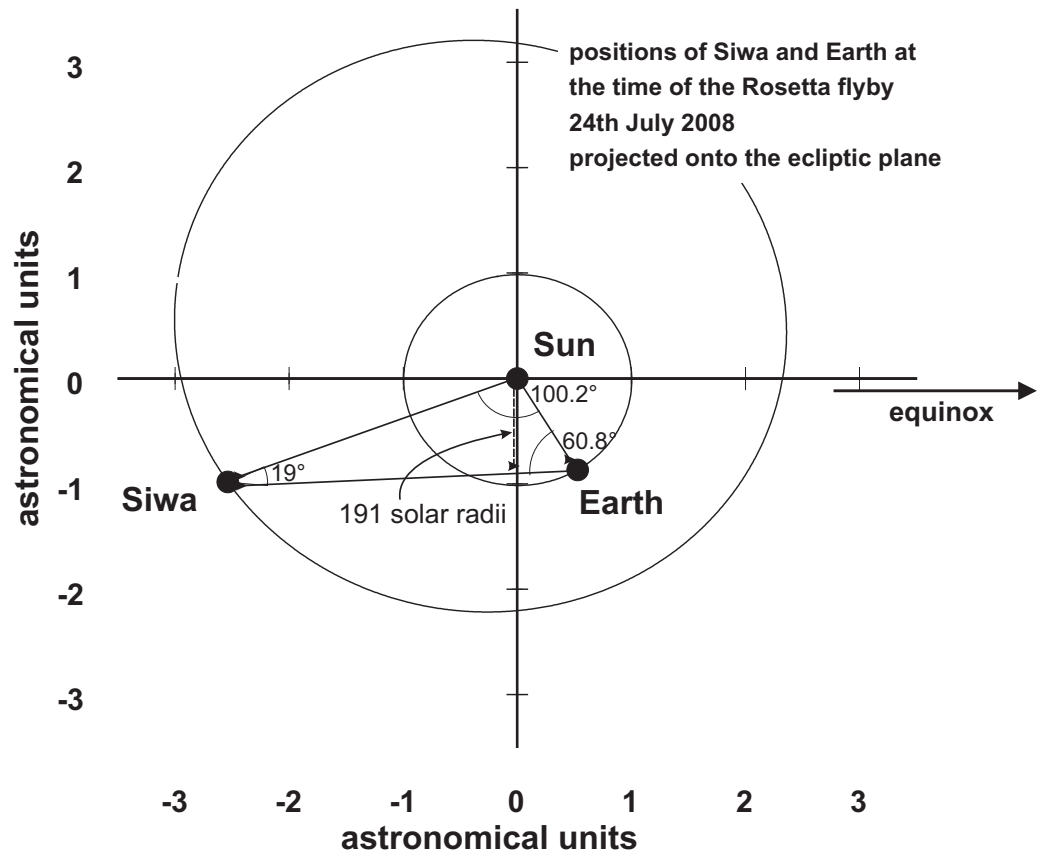

Fig. 5. Orbit positions of asteroid 140 Siwa and the Earth at the time of the Rosetta flyby (24th July 2008) projected onto the ecliptic plane. The angle between the heliocentric distance vectors toward Siwa and the Earth form an angle of $100^{\circ}$. The impact parameter of the radio ray path (closest approach of the radio ray to the Sun) is about 191 solar radii

\section{Summary}

The procedure of mass and bulk density determination of asteroids during spacecraft flybys was demonstrated and applied to the simulation of the Rosetta flyby at asteroid 140 Siwa on 24th July 2008. It is expected that the $\mathrm{RSI}$ radio science experiment on board Rosetta is able to determine the mass and bulk density of Siwa to an accuracy of $1 \%$ and $20 \%$, respectively, for the nominal flyby distance of $3500 \mathrm{~km}$ and flyby velocity of $17 \mathrm{~km} \mathrm{~s}^{-1}$

Attempts to determine the mass of the other Rosetta asteroid, 4979 Otawara, are unfortunately hopeless. Due to its small size (about $4 \mathrm{~km}$ ) and the large flyby distance $(1595 \mathrm{~km})$, the expected Doppler frequency is far below the noise level.

Acknowledgements. We would like to thank E. Gill and O. Montenbruck for their contributions to the experiment over the first four years and Marcello Fulchignoni for first-hand information on the asteroids Siwa and Otawara. The RSI experiment is funded by the Deutsches Zentrum für Luft- und Raumfahrt (DLR), Bonn, Germany, and by the National Aeronautics and Space Administration (NASA), Washington, USA.

\section{References}

Anderson, J. D., Armstrong, J. W., Campbell, J. K., et al. 1992, Space Sci. Rev., 60, 591
Anderson, J. D. 1971, Feasibility of Determining the Mass of an Asteroid from a Spacecraft Flyby, in Physical Studies of Minor Planets, ed. T. Gehrels, NASA SP-267, Washington D.C., U.S. Government Printing Office, 577

Anderson, J. D., \& Giampieri, G. 1999, Icarus, 138, 309

Bird, M. K. 1982, Space Sci. Rev., 33, 99

Belton, M., Chapman, C., Thomas, P. et al. 1995, Nature, 374, 785

Danby, J. M. A. 1992, Fundamentals of Celestial Mechanics, Second Edition (Willmann-Bell, Richmond, USA)

Doressoundiram, A., Weissman, P. R., Fulchignoni, M., et al. 1999, A\&A, 352, 697

Pätzold, M., Bird, M. K., Volland, H., Edenhofer, P., \& Buschert, H. 1991a, Z. Flugwiss. Weltraumforsch., 15, 89

Pätzold, M., Bird, M. K., Volland, H., Edenhofer, P., \& Buschert, H. 1991b, Z. Flugwiss. Weltraumforsch., 15, 159

Pätzold, M., Bird, M. K., Edenhofer, P. 1993, J. Geophys. Res., 98, A12, 20911

Pätzold, M., Neubauer, F. M., Wennmacher, A. et al. 2001, Rosetta Radio Science Investigations, ESA-Special Publication, in press

Rappaport, N. J., Giampieri, G., \& Anderson, J. D. 2000, Icarus, accepted for publication

Yeomans, D. K., Barriot, J.-P., Dunham, D. W., et al. 1997, Science, 278, 2106

Yeomans, D. K., Antreasian, P. G., Cheng, A., et al. 1999, Science, 285, 560

Yeomans, D. K., Antreasian, P. G., Barriot, J.-P., et al. 2000, Science, 289, 2085 